\title{
Eating Disorders in College Students in Iceland
}

\author{
Gudlaug Thorsteinsdottir, MD \\ Lilja Ulfarsdottir, PhD \\ Department of Psychiatry, Landspitali \\ University Hospital, Reykjavik
}

ICELAND

\begin{abstract}
Background and Objectives: The prevalence of eating disorders in Iceland is unknown. The purpose of this study was to estimate the prevalence of eating disorders in a large sample of college students in Iceland.

Methods: A sample of 3.052 students from around the country aged 15-20 years was used to determine prevalence of eating disorders. The Eating Disorders Diagnostic Scale (EDDS) and Eating disorder Screen for Primary care (ESP) were employed.

Results: On the ESP, $51.3 \%$ of females and $22.9 \%$ of males report discontent with their eating patterns and $63 \%$ of the females and $30.9 \%$ of the males report that they are emotionally affected by their weight. The ESP returned $10.5 \%$ prevalence when cut off level of 3 responses in the direction of an eating disorder was used, and 20.3\% when cut off level of 2 was applied. A total of $9.8 \%$ of participants received diagnosis with EDDS, $15.2 \%$ of females and $1.9 \%$ of males. For anorexia nervosa $1.1 \%$ of females received a diagnosis but no male. For bulimia nervosa $5.6 \%$ of females and $0.8 \%$ of males received a diagnosis and for binge eating disorder $0.6 \%$ of females and $0.2 \%$ of males. Prevalence of all subthreshold diagnoses combined was $5 \%$.

Conclusions: The prevalence of eating disorders is high in college students in Iceland, bulimia nervosa being the most common diagnosis for both males and females.
\end{abstract}

Received 10 July 2007

Revised 1 May 2008

Accepted 23 June 2008

\section{Introduction}

Eating disorders (ED) are of growing concern in the Western world. These disorders have become a relatively common psy- chiatric problem, especially among young women and adolescent girls. Studies reveal that lifetime prevalence of any eating disorder (ED) is at least $10 \%$ in adult women ${ }^{1}$. Many claim that eating disorders are on the rise but few studies have been published to verify 
this. Recent studies have focused on high prevalence of eating pathology among adolescents and college students in Western countries $^{2}$. Specifically, abnormal eating and weight control practices have been reported to be $26 \%$ for female and $10 \%$ for male college students ${ }^{3}$. In spite of this, little research has been done on the actual prevalence of EDs amongst college students and the vast majority of studies on the prevalence of EDs are on females only.

For anorexia nervosa (AN), the prevalence for young women has been reported to be $0.3 \%$ (range $0-0.9 \%)^{4}$ while the prevalence of bulimia nervosa $(\mathrm{BN})$ has been found to be in the range of $0.3-7.3 \%$ in females in Western countries 5 . For males, there is some research to suggest that the prevalence of $\mathrm{BN}$ among adolescent boys and adult men is $0.2 \%{ }^{6}$ but community studies on anorexia nervosa among males are practically nonexistent. The age of onset of ED is typically during adolescence ${ }^{7}$ but for binge eating disorder (BED) age of onset is more complicated. According to Reas and Grilo, patients seeking treatment for BED report different ages of onset of binge eating varying from 15 to 28 years of age, depending on the course of development of their disorder ${ }^{8}$. The prevalence of BED has been reported 1\% among 35 year old male and female participants ${ }^{9}$. The prevalence among adolescents appears to be much lower, but few studies are available. There is growing literature on eating disorders not otherwise specified (EDNOS), also referred to as subthreshold eating disorders, but general consensus regarding definitions is lacking which makes research difficult. It appears that EDNOS accounts for two thirds of all community cases with EDs and appears to be the most common ED diagnosed in specialized treatment settings ${ }^{10}$.

Iceland is a country with a population of about 300,000 people. It is a typical Western country with high living standards in general and urban society. EDs have become increasingly prominent in Iceland in the last few years. There has been a dramatic increase in referrals of cases to the health care sector with demands for specialized treatment. However, there are no published population based studies on the prevalence of ED in Iceland and services are underdeveloped. The only study on epidemiology of EDs examined the incidence of EDs in psychiatric inpatients in Iceland between 1983-2001 ${ }^{11}$. The results showed a significant increase of ED diagnoses in the specified time period, mostly due to more EDNOS and $\mathrm{BN}$ diagnoses while AN diagnoses remained fairly stable.

The aim of this study is to determine the prevalence of eating disorders amongst college students in Iceland. This study is the first community based research on the prevalence of EDs in Iceland.

\section{Methods}

\section{Sample}

In order to gather a large sample size it was decided to invite all major continuing education colleges in Iceland $(\mathrm{N}=28)$, including trade colleges, to participate in the study. These colleges provide 4 year preparatory studies for University. The principals of these colleges were contacted with a formal letter of invitation including a debriefing about the importance of the study. Nine large colleges in Reykjavik and vicinity participated and seven smaller colleges from different parts of the country. Of those colleges that did not participate five were from Reykjavik and seven from other parts of the country, mainly the larger towns. The colleges that declined participation did not differ from the participating colleges other than they were more likely to 
be located in the country. The reasons for not participating were that the schools thought too much effort was required for the study procedure and there were other ongoing studies in the college at the same time. The final sample consisted of 16 colleges around the country. In total, eighty two percent of participants came from Reykjavik and vicinity, but at the time of data collection a total of $63 \%$ of the population in the country was living in the greater Reykjavik area. The response rate varied between colleges from 55 to $99 \%$, with a mean response rate of $74.5 \%(\mathrm{SD}=17.83)$. The difference in response rate between the sexes was not considered.

The sample consisted of 3,052 students (1,234 males and 1,818 females) aged between 15 and 20 years (i.e. born between 1984 and 1988), Mean age was 17.75 (SD 1.16). This sample represents up to $22.18 \%$ of children born in Iceland during a particular year, averaging $14.56 \%$ of the children born during the years 1984 to 1988 .
Body mass index (BMI) was self reported and is included in the Eating Disorder Diagnostic Scale. The average BMI for the whole sample was $22.0(\mathrm{SD}=3.5)$ for the females and $23.0(\mathrm{SD}=3.4)$ for the males with an overall average of 22.4 ( $\mathrm{SD}=3.4)$. If we define normal weight as BMI between 18.5 and 25 then $76.8 \%$ of the males and $73.4 \%$ of the females are within normal weight range. See Figure 1.

\section{Assessment instruments}

Eating disorder screen for primary care (ESP)

$\mathrm{ESP}^{12}$ is a screening instrument for eating disorders which is composed of 5 questions. Two questions have been shown to be very sensitive towards EDs when posed by primary care physicians. Those questions are: "Do you ever eat in secret?" and "How satisfied are you with your eating habits?"13. These items are included in the ESP which is reported to have $100 \%$ sensitivity with 2 or

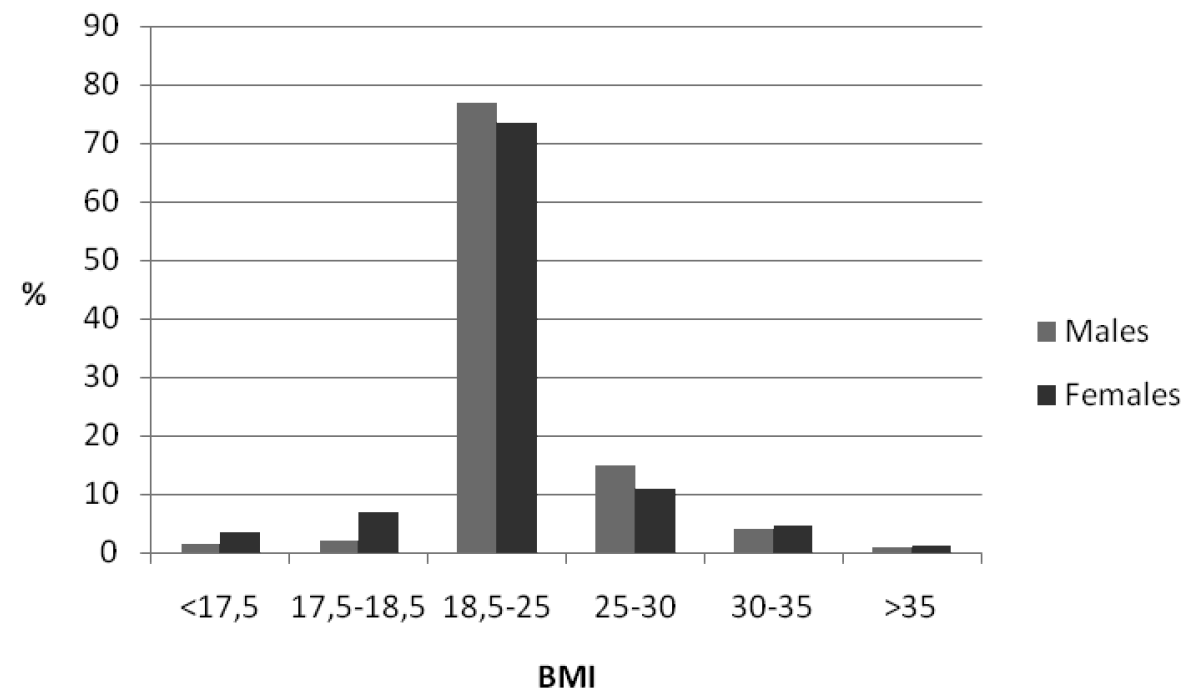

Figure 1. Distribution of the Body Mass Index of participants in percentage. This figure presents the BMI distribution for males $(\mathrm{n}=1235)$ and females $(\mathrm{n}=1771)$ 
more abnormal responses and $71 \%$ specificity for an ED. Three or more abnormal responses have been shown to rule in one ED, among a sample of university students and patients in primary care. The ESP was chosen because it is brief and provides a convenient comparison measure to the EDDS while not making the questionnaire too long.

The Eating Disorder Diagnostic Scale (EDDS)

EDDS is a 22-item questionnaire that returns ED diagnoses based on DSM-IV ${ }^{14}$. This instrument was adapted from the validated structured psychiatric interview: The Eating Disorder Examination (EDE) ${ }^{15}$ and the eating disorder module of the Structured Clinical Interview for DSM-IV (SCID) ${ }^{16}$.

Two psychometric studies have shown good reliability and validity of EDDS in general ${ }^{17}$, although the criterion validity for AN and BED is not well established. The instrument is developed on college women and has not been validated for men. The main benefits of using the EDDS are that it is quick and easy to administer and it was developed for college students. The EDDS has been validated in a small study in Iceland against the Bulit-R (Bulimia TestRevised) and an unstructured clinical interview ${ }^{18}$.

The EDDS returns both fullthreshold and subthreshold diagnoses for AN, BN and BED. A special feature of the EDDS is a continuous eating disorder symptom composite score (EDDSSYM) which is a summary of standardized scores (to control for different format of responses), indicating summary of overall ED symptoms. The EDDSSYM has been shown to correlate positively with validated measures of eating pathology ${ }^{14}$.

Both instruments were translated into Icelandic and back translated by experts prior to the study. Cronbach's alpha for the translated version of the EDDS for the symptom composite was 0.90 , standard.

\section{Procedure}

The study protocol was approved by the National Bioethics Committee and the Icelandic Data Protection Authority. The study was conducted from January 2004 to march 2005. Informed, written consent was obtained from all participants. This was collected in advance for participants under the age of 18 years, signed by parent or legal guardian. Other participants returned the signed consent form with the questionnaires. The students were asked to mark the questionnaires with identification number or year of birth. The procedure was thus not anonymous. The questionnaires were delivered in class by teachers who had been debriefed about the administration and consent forms. Participants returned the questionnaires in a sealed envelope which was provided with the questionnaires.

The prevalence is reported in terms of percentages. The ESP screen is correlated with the EDDSSYM score from the EDDS applying Pearson's $r$ correlation coefficient. Difference between EDDSSYM score of the specific diagnostic groups according to the EDDS is examined applying one way ANOVA and Turkey's HSD post hoc comparison. SPSS statistical package was applied for statistical analysis.

\section{Results}

A total of $10.5 \%(n=321)$ of the participants score 3 or more responses in the direction of ED on the ESP. Distribution of 
abnormal responses on individual questions of the ESP are presented in Table I. The most common abnormal response for both sexes is to the third and the first question respectively, where $63.1 \%$ of the females and $30.9 \%$ of the males say yes to question 3: Does your weight affect the way you feel about yourself? Similarly, $51.3 \%$ of females and $22.9 \%$ of males say no to question 1 : Are you satisfied with your eating patterns? If a cut off at 2 is used for the ESP to receive the maximum sensitivity, then $20.3 \%$ of the participants (26.2\% females, $11.1 \%$ males) may have an ED, but the specificity is low.

Results revealed a prevalence of ED as measured by EDDS of $9.8 \%(n=298)$. Specifically, the total prevalence of AN is $0.7 \%(n=20), \mathrm{BN}$ is $3.7 \%(n=112)$, BED is
$0.4 \%(n=13)$, and subthreshold diagnosis $5 \%(n=152)$. The ratio of females-males receiving ED diagnosis were 10/0,8. The most common diagnosis amongst the females is $\mathrm{BN}$ and subthreshold $\mathrm{AN}$ but for the males the most common diagnosis is $\mathrm{BN}$ and subthreshold BN.

Of the 298 participants that meet some diagnostic criteria on the EDDS there are only 22 males. No male participant received the diagnosis of AN. The results are detailed in Table II.

The EDDSSYM score from the EDDS measure is a sum of all items in the questionnaire and measures overall eating disorder symptoms. The EDDSSYM correlates with the $\operatorname{ESP}(r=0,684, p<0.01, n=3,004)$ which suggests the two are measuring the

Table I

A comparison of male and female abnormal responses on individual questions of the ESP. ( $N=3004)$

\begin{tabular}{lrr} 
ESP questions & \multicolumn{2}{c}{ Abnormal response } \\
& Females & Males \\
\hline Are you satisfied with your eating patterns? & $51.3 \%$ & $22.9 \%$ \\
Do you ever eat in secret? & $20.2 \%$ & $8.0 \%$ \\
Does your weight affect the way you feel about yourself? & $63.1 \%$ & $30.9 \%$ \\
Have any members of your family suffered with an eating disorder? & $12.8 \%$ & $7.9 \%$ \\
Do you currently suffer with or have you ever suffered in the past & $9.8 \%$ & $2.1 \%$ \\
$\quad$ with an eating disorder? & &
\end{tabular}

Note: ESP= Eating disorder Screen for Primary Care.

Table II

Prevalence of eating disorders according to EDDS $(\mathrm{N}=3052)$

\begin{tabular}{lrcr} 
EDDS Diagnosis & Girls & Boys & Total \\
\hline No diagnosis & $84.8 \%$ & $98.2 \%$ & $90.2 \%$ \\
Anorexia Nervosa & $1.1 \%$ & 0 & $0.7 \%$ \\
Bulimia Nervosa & $5.6 \%$ & $0.8 \%$ & $3.7 \%$ \\
Binge Eating Disorder & $0.6 \%$ & $0.2 \%$ & $0.4 \%$ \\
Subthreshold AN & $5.0 \%$ & $0.2 \%$ & $3.0 \%$ \\
Subthreshold BN & $2.6 \%$ & $0.6 \%$ & $1.8 \%$ \\
Subthreshold BED & $0.3 \%$ & $0.1 \%$ & $0.2 \%$ \\
Total with diagnosis & $15.2 \%$ & $1.8 \%$ & $9.8 \%$ \\
\hline
\end{tabular}

Note: EDDS = Eating Disorder Diagnostic Scale; AN = anorexia nervosa; $\mathrm{BN}=$ bulimia nervosa BED = binge eating disorder. 
same thing. However, the correlation is not perfect although significant which suggests that there are differences between the two measures.

A one way ANOVA revealed significant difference between the EDDS diagnostic groups on $\operatorname{EDDSSYM~}(F(6,3041)=255.20$, $p<0.001)$. However, Turkey's HSD post hoc comparison revealed that the EDDSSYM score does not show significant difference between participants who receive no diagno- sis and those with AN or subthreshold AN but for all the other diagnostic groups the difference between no diagnosis and diagnosis is highly significant $(p<0.001)$. These results are depicted in Figure 2. The EDDSSYM scores of AN, subthreshold AN and no diagnosis range from 11 to 13 . This does not represent a significant difference. For other diagnostic groups, which all involve binge eating, the scores are significantly higher, starting at 29 and peak at 46 for $\mathrm{BN}$.



Figure 2. Comparison of continuous eating disorder symptom composite (EDDSSYM) scores of different diagnostic groups. Note: subthr. $\mathrm{AN}=$ subthreshold anorexia nervosa; $\mathrm{AN}=$ anorexia nervosa; subthr.BED = subthreshold binge eating disorder; subthr. $\mathrm{BN}=$ subthreshold bulimia nervosa; $\mathrm{BED}=$ binge eating disorder; $\mathrm{BN}=$ bulimia nervosa.

\section{Discussion}

To our knowledge this is the largest study conducted on the prevalence of EDs in college students including both sexes. This study shows a high prevalence of ED in college students in Iceland with an overall prevalence of $9.8 \%$ as assessed with EDDS, $15.2 \%$ of the females received some ED diagnoses and $1.8 \%$ of the males. The high prevalence as measured with EDDS is supported by the ESP screening tool. Regarding the ESP, the most noticeable results are the high number of participants that score positively on the first and the third question. In spite of the majority of the sample being within normal weight range more than $50 \%$ of the women report dissatisfaction with their eating patterns and influence of their 
weight on self-esteem. This may reflect a normative discontent for young women in Western societies rather than actual eating disturbance. The high level of dissatisfaction among the males was unexpected but may be explained by that males are also experiencing much pressure to be thin and eat "healthy" foods. These results are cause for concern since body image problems and abnormal eating patterns are well known risk factors for the development of EDs ${ }^{19}$. Since this is the first study of EDs in a nonclinical sample in Iceland it is impossible to draw any conclusions about whether EDs are increasing in Iceland, thus further studies are needed.

The mean response rate of $74.5 \%$ was quite good, but there were significantly fewer males than females that participated in the study. This suggests that the response rate for the males was considerably lower than for the females since the gender ratio in Icelandic colleges is fairly even. We can only speculate about the reasons for this difference. It is possible that EDs are stigmatized by males and therefore they refuse to participate. Alternatively, it is possible that the males who did not participate are the ones who suffer some sort of ED and don't want to disclose their symptoms. However, 1235 males did take part which is to our knowledge the largest sample size reported in studies on ED in young males.

Regarding other comparative studies on college students in Western countries we could find no recent studies. The closest one for comparison is a study where the Survey for Eating Disorders (SED) questionnaire was used to assess ED in 14 and 15 year old adolescents (total 1960 boys and girls) in Norway. Results revealed lifetime prevalence for ED of $17.9 \%$ for girls, and $6.5 \%$ for boys which is also very high ${ }^{20}$ although lifetime prevalence studies always return higher numbers than point prevalence studies. Other surveys done in Norway and Sweden on ED in the general female population have reported a much lower prevalence of $3.8 \%$ and $3.2 \%$ respectively ${ }^{21,22}$. The comparatively high prevalence in the present study is possibly due to different assessment tools applied in the studies, different age groups and finally this may reflect that eating disorders are actually on the rise in the community.

In this study the prevalence of AN among the females is shown to be $1.1 \%$ which is in line with other studies. However, the EDDS did not diagnose any males with AN and only two males received diagnosis of subthreshold AN. It was therefore decided to look closer at the data for males with a $\mathrm{BMI}<17.5$, a total of 20 males. Of those 20 underweight males, 3 were found who scored high on questions 1-4 on the EDDS (which refer to fear of fatness, body image disturbance and the influence of weight on self-evaluation) as well as scoring 3 or more on the ESP. It is reasonable to suspect that these 3 males have a fullthreshold AN. However, on the computer scoring, 2 of these males received $\mathrm{BN}$ diagnosis and one received no diagnosis. If these males do have full threshold AN, then $0.3 \%$ of the males in the sample had full threshold AN which confirms the notion that AN is a very rare disorder among young males. The failure of the EDDS to recognize these males may be due to the fact that it is developed on women only.

Regarding BED and subthreshold BED, only $0.6 \%$ of participants receive a diagnosis with the EDDS and very few males. This may be explained by the young age of the participants or that the EDDS doesn't discriminate well enough between the $\mathrm{BN}$ and BED diagnosis, the binge eating behaviour being central to diagnosis of both disorders. 


\section{Limitations}

The most obvious limitation of this study is that it is not a two stage survey which is the ideal way to detect cases of any disorder with maximum amount of certainty. This is not to say that self report questionnaires should not be used, they can be extremely useful screening instruments for the "golden standard" clinical interview. In comparing interview and questionnaire data from a long-term follow-up study of BN, Keel et $a l .{ }^{23}$ conclude that questionnaires may not be inferior to interview assessments but reveal different aspects of eating pathology as anonymity may facilitate disclosure of symptoms ${ }^{23}$. For the purpose of the present study it was decided to use questionnaire for gathering data in order to maximize the number of participants. The EDDS was chosen since it returns ED diagnoses and not unspecific questions about body image and attitudes towards food. The draw backs of using the EDDS must be acknowledged here and they are mainly twofold. Firstly it is a relatively new instrument which poses problems with reference material and lack of studies for comparison. Secondly, while the benefits of using the EDDS were to have an instrument developed for college students, it does have the drawback of being developed on females only and may misdiagnose the males. Finally, it must be underscored that this study is limited to college students only so results cannot be generalized to the whole population.

In summary, eating disorders are frequent among college students in Iceland. The sex ratio being: 10 females/ 0.8 males. The authors recommend therefore a systematic screening for ED in primary care and colleges. Further studies employing diagnostic interviews are needed to replicate these findings and verify the ED diagnoses.

\section{Acknowledgements}

This project was sponsored by the Landspitali University hospital research foundation. Wyeth-Lederle, Lilly, AstraZeneca also supported this study. The authors would also like to thank all the participants and the college officials who made this study possible, for their contribution, positive attitude and understanding of the importance of the study.

\section{References}

1. Stice E, Shaw H, Burton E, Wade E. Dissonance and healthy weight eating disorder prevention programs: a randomized efficacy trial. J Consult Clin Psychol 2006 Apr; 74(2): 263-275.

2. Hoerr SL, Bokram R, Lugo B, Bivins T, Keast DR. Risk for disordered eating relates to both gender and ethnicity for college students. J Am Coll Nutr 2002 Aug; 21(4): 307-314.

3. Forman-Hoffman V. High prevalence of abnormal eating and weight control practices among U.S. highschool students. Eat Behav 2004 Nov; 5(4): 325-336.

4. Hoek HW, van Hoeken D. Review of the prevalence and incidence of eating disorders. Int J Eat Disord 2003 Dec; 34(4): 383-396.

5. Makino M, Tsuboi K, Dennerstein L. Prevalence of eating disorders: a comparison of Western and non-Western countries. Med Gen Med 2004; 6(3): 49.

6. Carlat DJ, Camargo CA, Jr., Herzog DB. Eating disorders in males: a report on 135 patients. Am J Psychiatry 1997 Aug; 154(8): 1127-1132.

7. Kohn M, Golden NH. Eating disorders in children and adolescents: epidemiology, diagnosis and treatment. Paediatr Drugs 2001; 3(2): 91-99.

8. Reas DL, Grilo CM. Timing and sequence of the onset of overweight, dieting, and binge eating in overweight patients with binge eating disorder. Int J Eat Disord 2007 Mar; 40(2): 165-170.

9. Hay P. The epidemiology of eating disorder behaviors: an Australian community-based survey. Int J Eat Disord 1998 May; 23(4): 371-382. 
10. Machado PP, Machado BC, Goncalves S, Hoek HW. The prevalence of eating disorders not otherwise specified. Int J Eat Disord 2006 Dec 15.

11. Thorsteinsdottir G. Eating disorders at Landspitali University Hospital, Reykjavik 1983-2001. Laeknabladid 2003 August; supplement 48S: 62.

12. Cotton MA, Ball C, Robinson P. Four simple questions can help screen for eating disorders. J Gen Intern Med 2003 Jan; 18(1): 53-56.

13. Freund KM, Graham SM, Lesky LG, Moskowitz MA. Detection of bulimia in a primary care setting. J Gen Intern Med 1993 May; 8(5): 236-242.

14. Stice E, Telch CF, Rizvi SL. Development and validation of the Eating Disorder Diagnostic Scale: a brief self-report measure of anorexia, bulimia, and binge-eating disorder. Psychol Assess 2000 Jun; 12(2): 123-131.

15. Cooper Z, Cooper PJ, Fairburn CG. The validity of the eating disorder examination and its subscales. Br J Psychiatry 1989 Jun; 154: 807-812.

16. Spitzer RL, Williams JB, Gibbon M, First MB. The Structured Clinical Interview for DSM-III-R (SCID). I: History, rationale, and description. Arch Gen Psychiatry 1992 Aug; 49(8): 624-629.

17. Stice E, Fisher M, Martinez E. Eating disorder diagnostic scale: additional evidence of reliability and validity. Psychol Assess 2004 Mar; 16(1): 60-71.

18. Jonsdottir SM, Thorsteinsdottir G, Smari J. [Reliability and validity of the Icelandic version of the Bulimia
Test-Revised (BULIT-R)]. Laeknabladid 2005 Dec; 91(12): 923-928.

19. Chamay-Weber C, Narring F, Michaud PA. Partial eating disorders among adolescents: a review. J Adolesc Health 2005 Nov; 37(5): 417-427.

20. Kjelsas E, Bjornstrom C, Gotestam KG. Prevalence of eating disorders in female and male adolescents (14-15 years). Eat Behav 2004 Jan; 5(1): 13-25.

21. Gotestam KG, Agras WS. General population-based epidemiological study of eating disorders in Norway. Int $\mathrm{J}$ Eat Disord 1995 Sep; 18(2): 119-126.

22. Ghaderi A, Scott B. Prevalence, incidence and prospective risk factors for eating disorders. Acta Psychiatr Scand 2001 Aug; 104(2): 122-130.

23. Keel PK, Crow S, Davis TL, Mitchell JE. Assessment of eating disorders: comparison of interview and questionnaire data from a long-term follow-up study of bulimia nervosa. J Psychosom Res 2002 Nov; 53(5): 10431047.

Address for correspondence:

Gudlaug Thorsteinsdottir, MD

Department of Psychiatry

Landspitali University Hospital

Hringbraut

101 Reykjavik

ICELAND

Phone: (354) 543-1000

Fax: (354) 5434816

E-mail: gudlthor@landspitali.is 